\title{
Semantic Text Classification of Emergent Disease Reports
}

\author{
Yi Zhang and Bing Liu \\ Department of Computer Science, University of Illinois at Chicago, \\ 851 S. Morgan Street, Chicago IL 60607, USA \\ \{yzhang 3,1 iub\}@cs.uic.edu
}

\begin{abstract}
Traditional text classification studied in the information retrieval and machine learning literature is mainly based on topics. That is, each class represents a particular topic, e.g., sports and politics. However, many real-world problems require more refined classification based on some semantic perspectives. For example, in a set of sentences about a disease, some may report outbreaks of the disease, some may describe how to cure the disease, and yet some may discuss how to prevent the disease. To classify sentences at this semantic level, the traditional bag-of-words model is no longer sufficient. In this paper, we study semantic sentence classification of disease reporting. We show that both keywords and sentence semantic features are useful. Our results demonstrated that this integrated approach is highly effective.
\end{abstract}

Keywords: Semantic text classification.

\section{Introduction}

In traditional topic-based text classification, the bag-of-words representation of text documents is often sufficient because a topic can usually be characterized by a set of keywords $[12,13]$. However, for semantic classification, the n-gram representation is no longer sufficient because the texts from different classes may be on the same topic. To classify texts at such a level, the system needs to capture some semantic characteristics in order to perform more accurate classification.

In this paper, we propose to integrate the bag-of-words scheme and semantic features extracted from sentences for classification. As a case study, we investigate the disease reporting domain. We want to classify sentences that report disease outbreaks, and sentences that do not. For example, the following sentence reports a possible disease outbreak "the district hospital reported today that 10 people were diagnosed with cholera this morning". However, the following sentence does not report an outbreak, "the district hospital reported today that they have successfully tested a new cholera treatment procedure". Both sentences are on the topic of cholera. However, they are entirely different semantically. The problem is how to separate sentences based on the required semantic categories, i.e., reporting a possible outbreak or not in this case. We note that sentences rather than documents are used in this work because a document contains a large number of sentences and the sentences have quite different semantic meanings. For example, a piece of disease outbreak 
news may contain many pieces of other related information, e.g., symptoms, treatment, vaccine, and past disease history. We show that both the words used in sentences and the sentence semantic characteristics are important. Our experimental results confirm that this integrated approach produces more accurate classifiers than each of them alone. Another original work is the investigation of date representations in natural language.

\section{On Semantic Classification}

The setting of semantic classification is the same as traditional topic-based text classification. Given a set of documents $D$ and each document $d \in D$ is labeled with a class $c \in C$, where $C$ is a set of known classes. However, semantic classification usually has more refined classes, which are hard to be separated based on bag-ofwords or n-grams alone. For example, the sentence, "the district hospital reported that 10 people were diagnosed with cholera early today", reports a possible cholera outbreak. It is easy to observe that "reported" and "diagnosed" are indicative of an outbreak. The time "today" indicates a new event. Using the words alone, however, is insufficient as this sentence illustrates: " 10 people from the district hospital submitted a report early today on cholera diagnosis." This sentence uses very similar words, but has a completely different semantic meaning, i.e., no disease outbreak is reported.

In this paper, we define semantic information as any information extracted from the sentence that is not based on keywords/n-grams. There are multiple levels of semantic information: at the highest level it is the full understanding of the text, which is still not possible with the state-of-art technology; at lower levels, we have features with different amounts of semantic contents, extracted from sentences using current NLP techniques. The exact features used in this work will be discussed in the next section.

We note that the bag-of-words scheme is still useful because to express a particular semantic meaning, certain keywords are more likely to be used. Semantic feature extraction is still not perfect. Keywords can help offset some of the errors. Fig. 1 illustrates the difference between traditional text classification and semantic text classification as described in this work. Note that we do not make a difference of the types of classes or texts used in a classification task because classification of different types of categories may be assisted by some level of semantic information.

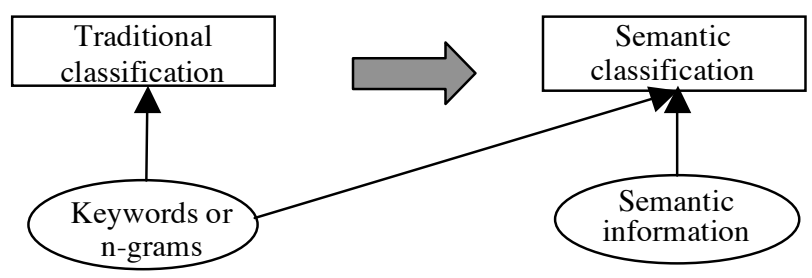

Fig. 1. Traditional classification and semantic classification 


\section{Important Semantic Features}

Our aim is to classify sentences that report possible disease outbreaks and those that do not, which is a classification problem. We will use a supervised machine learning algorithm, e.g., naïve Bayesian or support vector machines. Thus, we only need to design and construct features. As we mentioned above, we use both keywords and semantic features. Keyword features are obtained in the same way as in traditional text classification. Here, we only focus on semantic features.

\subsection{Noun Phrase Containing a Disease Word}

Center word of a noun phrase: The center word in a noun phrase has a more direct influence on the overall semantic meaning than any other word in the noun phrase. For example, the center word of the noun phrase "their new cholera treatment procedure" is "procedure". We only use noun phrases containing a disease word because such phrases are more likely to be relevant to our classification task.

Negation modifier and determiner word: Other important features in a noun phrase include negation modifiers e.g., "no", and determiner words such as "every". Their importance can be illustrated by the following examples: "No case of cholera has been found yesterday" indicates no disease, and "For every case of mad cow disease in Switzerland, 100 animals may carry the infection silently" gives a study result on the disease rather than a specific case.

\subsection{Verb Phrase}

Verb and adjective: The verb serves as the main skeleton of a sentence and thus is an important feature. Sometimes, a verb is too common to have a specific meaning. In that case, the adjective word after the verb becomes important. For example, the verbs "is" and "become" are not specific, but "is ill" and "become sick" are.

Tense: The verb tense is important for the semantic meaning of a sentence because it shows the time or the subjunctive mood. Past perfect tense usually means something happened in the long past. E.g., "West Nile Virus had plagued US" refers to an old event. Subjunctive mood expressed by the past-future perfect tense is often used for conjectures. E.g., "a bird flu outbreak could have killed millions of people" is a conjecture of the disease's impact rather than a report of an actual outbreak.

Other features extracted from a verbal phrase include: auxiliary word, verb phrase being an if-whether clause, negation word, verb phrase being an adjective clause, and subject/object.

\subsection{Dates}

Dates are important for disease reporting. We focus on the common ones in this work. Thus, our description below is by no means complete, but is quite sufficient for our data. Date information is usually expressed by a prep word (maybe omitted) followed by a date phrase. We call the date expressed in the text as the expressed date, and the date of the context as the context date (e.g., publication date of a news report). 
Prep word: A prep word decides the relationship between an expressed date and the date phrase that follows. We summarize the prep words and the corresponding relationships in Table 1. If a prep word is omitted, in most cases it's the same as the first relationship in the table. For example, "The alert was given last Tuesday".

Table 1. Prep words and relationships between expressed dates and date phrases

\begin{tabular}{|l|c|c|}
\hline \multicolumn{1}{|c|}{ Relationship } & Prep word & Example \\
\hline expressed date is the date phrase & in, at, on, during & on Monday \\
\hline expressed date is before the date phrase & before & before winter \\
\hline expressed date is after the date phrase & after & after May 1, 2006 \\
\hline expressed date ends within the date phrase & in, within & in two days \\
\hline expressed date ends by the date phrase & by, as of, until/till, no later than & by today \\
\hline expressed date spans the two date phrases & between ... and, from...to... & From Jan to Feb \\
\hline expressed date starts from the date phrase & since & since last year \\
\hline
\end{tabular}

Adjective and adverb: Adjectives and adverbs may be associated with dates, e.g., "ago" as in "three months ago". In general, a date phrase expresses either an absolute date or a relative date. We will not discuss time in this paper as it can be dealt with in a similar way.

Absolute date: As its name suggests, an absolute date expresses a specific date without ambiguity regardless when it is seen. There are two main types:

- Historic period: It is a time period in history usually with a very long duration, and it has a specific name, e.g., "Stone Age".

- Formal date: It specifies an absolute time period that can be: a century, a decade, a year, a season, a month, a day, a time period of a specific day, etc.

Relative date: Its absolute date can only be determined based on the context date.

- Recurrent named date: Such a relative date occurs repetitively, e.g., annual festival, season, month of year, day of month, and day of week. A restrictive modifier is mandatory, although sometimes it can be omitted based on convention.

- Other named dates: Such dates include "today" and "tomorrow" or special words (e.g., "now" and "recently") that are dedicated to some relative dates.

- Number-unit: This is also popularly used in date phrases, e.g., "three months" in "three months ago". Similar to a recurrent date, a modifier is also required.

A date phrase may have a refiner, such as "early 2007" and "the end of last month". Now we give a formal definition of date phrases in Backus-Naur form. Due to space limitations, some rules use suspension points in place of similar entries, and definitions of self-explanary items (e.g., $<$ DateUnit $>$ and $<$ Number $>$ ) are omitted.

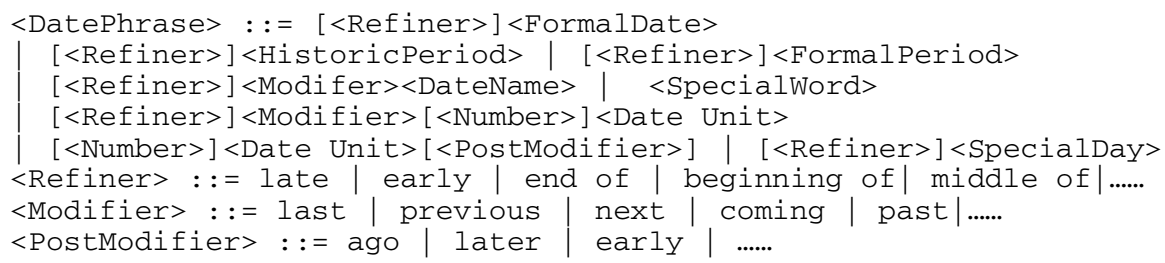




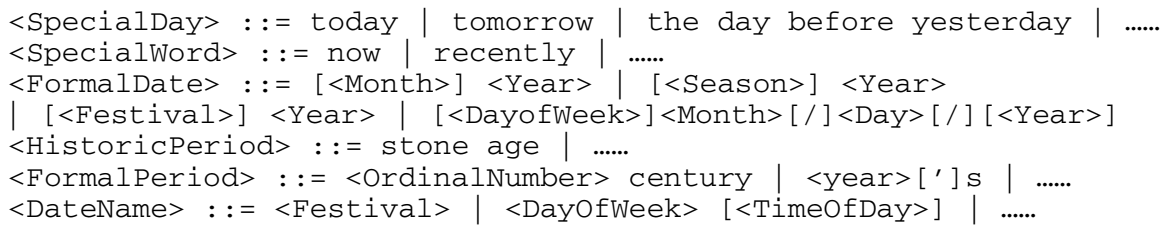

\section{Feature Extraction}

Now we describe how to extract features from a sentence. Our first task is to recognize named entities, because most features given in Section 3 can only be found based on correct recognition of named entities, i.e., disease names or dates.

\subsection{Dependency Tree}

A dependency tree describes the syntactical and semantic relationships in a sentence. Fig. 2 gives a dependency tree generated from the sentence "Danish health authorities on Friday confirmed the Scandinavian country's fourth case of mad cow disease".

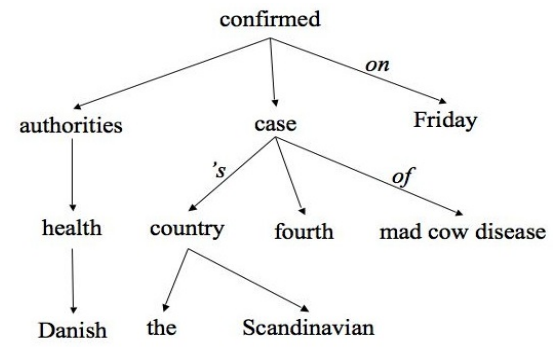

Fig. 2. An example of dependency tree. Preposition words and the word "s" are put on the edge to save space. Arrows point from a parent node to children nodes. Note that "mad cow disease" is a named entity, so it is a single node in spite of that it has three literal words.

\subsection{Feature Extraction and Construction}

After named entities have been recognized and the dependency tree has been built, features are extracted in the following way: Starting from any infectious disease, we find the center word of the noun phrase containing the disease. Negation modifier and determiner words can be found among children nodes of the center word.

The verb is always the nearest ancestral verb node of the noun phrase. If there is an adjective node between the verb and the noun phrase, it is taken as the adjective word feature. The tense of a verb is determined by the form of the verb and the forms of auxiliary words, which are children nodes of the center word. So are negation modifiers and subject/object words. Other features of a verb phrase can be obtained from sibling or parent nodes of the verb node. If a verb phrase is an adjective clause, the verb node normally has a sibling node of "wh-" word and a relationship of complementary to its parent node. If a verb phrase is an if-whether clause, there will 
be a sibling node of "if" or "whether". Date feature can be recognized using the definitions given in Section 3.3. To normalize dates, we treat a relative date in current year as "recent", any date before that as "old", and any date after as "future". Thus, the date feature has three possible values, "recent", "old" and "future".

Implementation: We use MINIPAR [7] for dependency tree generation and named entity recognition. In order to recognize diseases, we supplemented the standard MINIPAR database with disease names extracted from Centers for Disease Control and Prevention (http://www.cdc.gov/ncidod/). The feature construction algorithm then reads the generated dependency trees and outputs features.

\section{Experiments}

Experimental data: Our corpus consists of sentences related to infectious diseases. Some of them are emergent disease reports (EDR), and others are not (non-EDR) but still contain the disease names. The sentences are extracted from disease report documents in ProMED-mail (http://www.promedmail.org). We labeled the sentences into two classes: EDR(1660 sentences) and nonEDR (682 sentences).

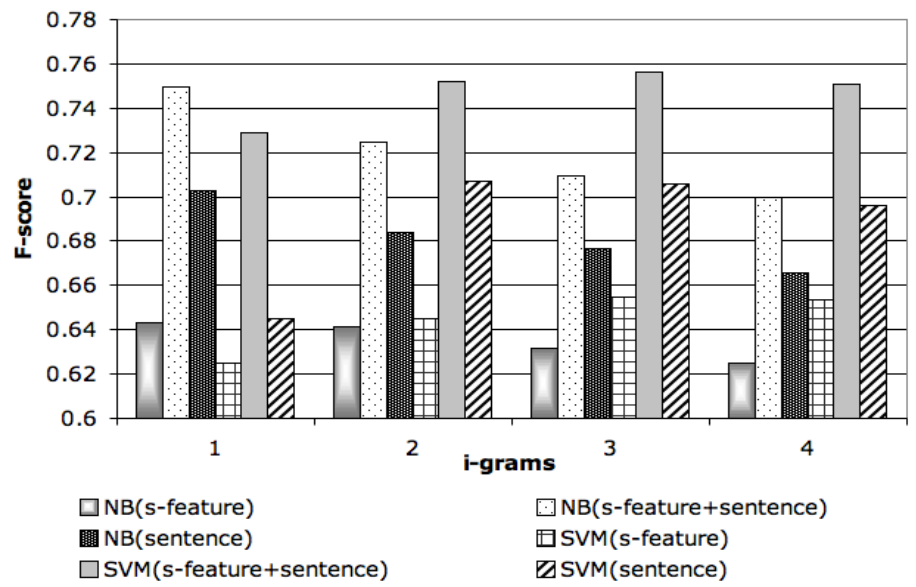

Fig. 3. Experimental results

Experimental settings: Two popular supervised learning algorithms are used to build models, Support Vector Machines (SVM) and naïve Bayesian (NB). Both algorithms are provided in the latest version of the Rainbow package [8], which is used in our experiments. Different types of features are employed and compared:

- sentence: bag-of-words representation with 1-gram, 2-gram, 3-gram and 4-gram.

- s-features: semantic features (including the date feature).

- s-features+sentence: s-features and sentence features in a sentence combined. 
To ensure reliable results, we run each technique 10 times. In each run, $80 \%$ of the data (randomly selected) is used for training and $20 \%$ of the data is used for testing. The results are then averaged and reported below. The evaluation measure is F-score on EDR sentences. F-score is the harmonic mean of precision $(p)$ and recall $(r)$, i.e., $F$ $=2 p r /(p+r)$, which is commonly used in text classification.

Results: Fig. 3 shows the average F-scores of all methods. We observe that semantic features (denoted by s-features) are very helpful. Both SVM and NB produce much better results when sentences and semantic features are both used. SVM (sfeatures+sentence) with 3-gram for sentences gives the best F-score and it also performs the best for 2-gram and 4-gram, except for 1-gram, in which NB (sfeatures+sentence) is better. Please note that we are applying i-grams for all three feature sets, so i-grams are also relevant for "s-feature" feature set, as a result, Fscores for NB (s-feature) and SVM(s-feature) differ across different orders of i-grams.

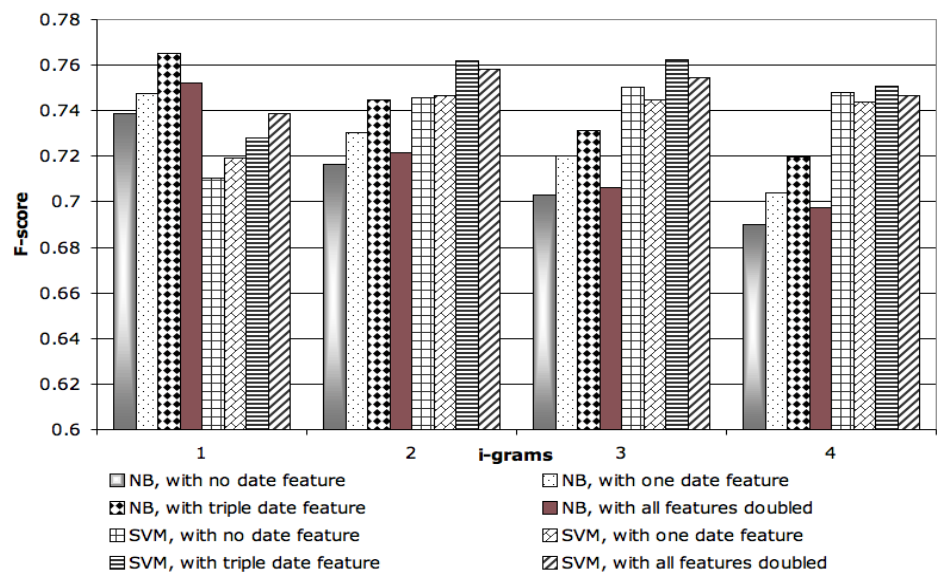

Fig. 4. Experimental results: with different weights of date feature

We also single out the date feature to see how it effects classification since intuitively the dates are important for EDR sentences. The date feature is indeed helpful (Fig. 4). For NB, the F-scores with date features are always better than without date features. For SVM, the results are also better for 1-gram and 2-gram. All the results here use both s-features and sentences. Since the date feature has shown its importance, it may help more if its weight is increased. We found that multiplying each date feature by 3 gives the best results. Fig. 4 shows that "triple date feature" gives better F-scores for both NB and SVM in almost all cases. NB with 1-gram produces the best result. Due to this success, we also tried to increase the weights of all s-features ("with all features doubled") but without improvements (Fig. 4).

In summary, we can conclude that combining bag-of-words and semantic features indeed improve classification. The date feature is also very helpful. 


\section{Related Work}

There are several works on using linguistic information for text classification. Most of them are based on the idea of carefully choosing keywords or phrases. Noun phrases have been used in [2], and terms features extracted from part-of-speech tags were used in [1]. [11] improved the text classification by using bag-of-concepts. [10] investigated adding complex nominals as features. [3] reported classification results based on several keyword extraction methods. [4] assigned feature weights based on the importance of each sentence determined by a text summarization system. Similar works were also reported in $[6,9]$. Sub-trees of dependency trees were shown helpful in classification in [5]. Our work is related but also different from these text classification works in several ways. First, they still focus on classifying whole text documents, but we focuses on sentence level classification, which requires more delicate semantic features. We also construct date features, which is also new.

\section{Conclusion}

In this paper, we studied the problem of classifying disease reporting at the semantic level. It is shown that both keywords and semantic features are valuable for the task. The paper also investigated the representation of dates, which will be useful to other applications. Experimental results demonstrated that the proposed integrated approach significantly outperforms each individual approach alone.

Acknowledgments. This work is funded by the Great Lakes Protection Fund. We also thank Karl Rockne for helpful discussions.

\section{References}

1. Aizawa, A.: Linguistic techniques to improve the performance of automatic text categorization. NLPRS-01 (2001)

2. Furnkranz, J., Mitchell, T., Riloff, E.: A case study using linguistic phrases for text categorization on the WWW. In: AAAI-98 Workshop on Learning for Text Categorization

3. Hulth, A., Megyesi, B.: A Study on Automatically Extracted Keywords in Text Categorization. In: ACL-06 (2006)

4. Ko, Y., Park, J., Seo, J.: Improving text categorization using the importance of sentences. Info. Proc. and Manag. 40(1), 65-79 (2004)

5. Kudo, T., Matsumoto, Y.: A boosting algorithm for classification of semi-structured text. In: EMNLP-2004 (2004)

6. Li, C., Wen, J.-R., Li, H.: Text classification using stochastic keyword generation. In: ICML-03 (2003)

7. Lin, D., Pantel, P.: Discovery of Inference Rules for Question Answering. Nat. Lang. Eng. 7(4) (2001)

8. McCallum, A.: Bow: A toolkit for statistical language modeling, text retrieval, classification and clustering (1996), http://www.cs.cmu.edu/ mccallum/bow 
9. Mihalcea, R., Hassan, S.: Using the essence of texts to improve document classification. In: RANLP-2005 (2005)

10. Moschitti, A., Basili, R.: Complex linguistic features for text classification: A comprehensive study. In: ECIR-04 (2004)

11. Sahlgren, M., Coster, R.: Using bag-of-concepts to improve the performance of support vector machines in text categorization. In: COLING 2004 (2004)

12. Sebastiani, F.: Machine learning in automated text categorization. ACM Computing Surveys 34(1), 1-47 (2002)

13. Yang, Y., Liu, X.: A re-examination of text categorization methods. In: SIGIR-99 (1999) 